\title{
The Coronavirus Flu Emergency in Italy: Legal Issues Regarding Minors
}

\author{
Piergiorgio Fedeli ${ }^{1}$, Nunzia Cannovo ${ }^{2}$, Roberto Scendoni ${ }^{3}$ and Mariano Cingolani ${ }^{3 *}$ \\ ${ }^{1}$ University of Camerino - School of Law, Institute of Legal Medicine, Italy \\ ${ }^{2}$ Etich committee, University of Naples
}

${ }^{3}$ Department of Law, Institute of Legal Medicine, University of Macerata, Italy

*Corresponding author: Mariano Cingolani, University of Macerata- Department of Law - Institute of Legal Medicine - Via Don

Minzoni n. 9, 62100, Macerata, Italy

\section{ARTICLE INFO}

Received: March 17, 2020

Published: 幽 April 03, 2020

\section{ABSTRACT}

The COVID 19 infection has been faced as an epidemic, through measures to enforce a high degree of isolation. These regulations hold for minors, as well, with consequent difficulties for this age group, which at the moment seems to be least vulnerable to the severe complications of COVID 19.

Citation: Fedeli P., Cannovo N., Scendoni R., Cingolani M. . The Coronavirus Flu Emergency in Italy: Legal Issues Regarding Minors. Biomed J Sci \& Tech Res 26(5)2020. BJSTR. MS.ID.004421.

\section{Introduction}

In 2017 in Italy, researchers identified a coronavirus cluster in bats in northern Italy, with peculiar genetic characteristics [1], but no further inquiry was conducted on its virulence in the hosts. The new coronavirus causes "severe acute respiratory syndrome coronavirus 2" (SARS-CoV-2). Propagation happens person-toperson through respiratory droplets transmitted when a patient sneezes or coughs [2]. At greatest risk of grave complications are those with chronic diseases and low immune response [3], including the elderly, while the symptomatology in children appears to be less grave.Transmission by asymptomatic subjects has not been excluded, and thus currently isolation is the first form of prevention [4]. Nevertheless, this information the infection from COVID-19 has been faced as an epidemic, through measures to enforce a high degree of isolation. This has entailed a complex management model with individual measures, structures and logistics aimed at avoiding the spread of the infection in the nosocomial sphere and consequently in the community.

\section{The Characteristics of COVID 2019 in Children}

Early in the outbreak, the National Respiratory Diseases Clinical Research Centre and Respiratory Diseases Group of the Chinese
Paediatric Association began a study on the clinical features of COVID 19 in paediatric patients, providing the first information on the characteristics of COVID 19 flu in children [5], which currently seems to be less severe than the clinical forms in adults [6]. One Chinese study indicated that children are seldom attacked by COVID 19 or SARS-CoV and suggested that this was due to the Chinese vaccination program for minors. In particular, "the RNA-virus vaccines and the adjuvants in vaccine programs may help children escape from the infection [7]". However, according to other authors, the number of paediatric patients suffering from COVID 19 "could rise in the future, and a lower number of paediatric patients at the beginning of a pandemic does not necessarily mean that children are less susceptible to the infection [8]". Complete understanding of the physiological mechanisms underlying the lower rate of infection among children could help in battling the diametrically opposite gravity of the infection in adults.

On the basis of experiences reported in China [9], the clinical manifestations of the COVID 19 infection in children are "fever (generally low or absent), fatigue and cough, nasal congestion, runny nose, expectoration, diarrhoea, headache, etc." After about a week of infection, children may develop "dyspnoea, cyanosis 
accompanied by systemic toxic symptoms, such as malaise or restlessness, poor feeding, bad appetite and less activity." There can also be "abdominal discomfort, vomiting, abdominal pain and diarrhoea". In the most severe cases, "septic shock, metabolic acidosis and irreversible bleeding and coagulation dysfunction may occur." "In the early phase of the disease, white blood cell count is normal or decreased, or with decreased lymphocyte count [10]. Radiological signs attest the presence of viral pneumonia at the beginning, which can advance to the point of signs of lung consolidation [11]. Kunling Shen et al. [12] described clinical classifications of the symptoms in minors are

a. Asymptomatic infection (silent infection);

b. Acute upper respiratory tract infection;

c. Mild pneumonia;

d. Severe pneumonia;

e. Critical cases.

To date (March 11, 2020), there have been no deaths of children infected with COVID 19 in China [13] or in Italy [14]. In fact, on the basis of current scientific evidence, it can be hypothesized that in children and young adults the infection presents few symptoms or is asymptomatic [15].

There has been no indication of vertical transmission from mother to infant, not even through maternal milk [16]. In fact, the Italian Society of Neonatology has proposed that a woman who has tested positive for corona virus but is asymptomatic should be allowed to nurse and be close to her newborn. Instead, if she shows symptoms of fever, coughing and respiratory secretions, she and her newborn should be separated, if she consents to this and if the logistics of the hospital permit it [17]. No standard treatment has been identified, and current treatment plans for children are adapted from those for adults [18]. Contact between medical personnel and patients must be kept to the minimum; visitors should have limited access and wear proper protective items [19]. In such a context, there is also need for psychological support [20].

\section{Care for Minor Patients in Italy}

The choice in Chinese healthcare protocols to isolate minors suffering from COVID 19 and to limit their contact with others seems quite problematic for an Italian setting. The situation in which a healthy parent desires to stay with a child sick with COVID 19 presents complex questions. Italian guidelines on precautionary isolation contain no explicit exceptions when the patient is a minor. However, in such a case, even if the parent uses personal protection devices to prevent contagion, allowing him or her to stay there may indicate a lack of proper attention to and protection of the health of the parent. Italian legislation recognizes the minor as a vulnerable subject for whom specific protections should be provided by parents or legal representatives, who are also required by law to care for the minor's health. Minors “must receive information about healthcare choices in a form appropriate to their ability to understand, so that they can express their wishes" (L. 219/17 art. 3). Informed consent to treatment for a minor is provided by parents or legal guardians [21,22].

When off label treatment is proposed, as may be the case with COVID 19, the weight of the parents' informed consent is even more important [23]. Clearly, the COVID 19 epidemic is a situation of emergency and experimentation. Can an emergency situation justify the administration of drugs of uncertain efficacy, especially when the patient is a minor? In addition, since what is at issue is essentially clinical experimentation, would it not be useful to request urgently the opinion of an Ethics Committee? Would it not be desirable to receive some direction from the Health Ministry? This would be valuable not only for minors, but for all gravely ill subjects who are incapable of expressing their own decision. Fortunately, COVID 19 has not generated dramatic clinical manifestations in minors, and Italy has not had to face these problematic issues in the management of paediatric patients. Other considerations not strictly of a healthcare nature concern the right to study and the risk of stigmatization and discrimination. It is evident that the restrictive regulations introduced with the most recent ministerial ordinances closing all schools and universities in Italy will undermine the right to study of minors, but in the interests of preventing possible COVID 19 infection, which in the long run may be the greater good.

\section{References}

1. Francesca Rizzo, Kathryn M Edenborough, Roberto Toffoli, Paola Culasso, Simona Zoppi, et al. (2017) Coronavirus and paramyxovirus in bats from Northwest Italy, BMC Veterinary Research13: 396.

2. Jasper Fuk Woo Chan, Shuofeng Yuan, Kin Hang Kok, Kelvin Kai Wang To, Hin Chu, et al. (2020) A familial cluster of pneumonia associated with the 2019 novel coronavirus indicating person-to-person transmission: a study of a family cluster. Lancet 395: 514-523.

3. Jaillon S, Berthenet K, Garlanda C (2019) Sexual dimorphism in innate immunity. Clin Rev Allergy Immunol 56: 308-321.

4. Marco Cascella, Michael Rajnik, Arturo Cuomo, Scott CDulebohn, Raffaela Di Napoli, et al. (2020) Features, Evaluation and Treatment Coronavirus (COVID-19). Stat Pearls Publishing.

5. KunLing Shen, Yong Hong Yang (2020) Diagnosis and treatment of 2019 novel coronavirus infection in children: a pressing issue. World Journal of Pediatrics.

6. Wang XF, Yuan J, Zheng YJ, Chen J, Bao YM, et al. (2020) Clinical and epidemiological characteristics of 34 children with 2019 novel coronavirus infection in Shenzhen17 58(0): E008.

7. Lei ZhangYunhui Liu (2020) Potential interventions for novel coronavirus in China: A systematic review. J Med Virol 92(5): 479-490.

8. PingIngLee, YaLiHub, PoYenChen, YhuChering Huang, PoRenHsueh, et al. (2020) Are children less susceptible to COVID-19? Journal of Microbiology, Immunology and Infection.

9. ZhiMin Che, Zhi Min Chen, Jun Fen Fu, Qiang Shu, Ying Hu Chen, et al. (2020) Diagnosis and treatment recommendations for pediatric respiratory infection caused by the 2019 novel coronavirus. World Journal of Pediatrics. 
10. Kunling Shen, Yonghong Yang, Tianyou Wang, Dongchi Zhao, Yi Jiang et al. (2020) Diagnosis, treatment, and prevention of 2019 novel coronavirus infection in children: experts' consensus statement. World Journal of Pediatrics.

11. Kunling Shen, Yonghong Yang, Tianyou Wang, Dongchi Zhao, Yi Jiang et al. (2020) Diagnosis, treatment, and prevention of 2019 nove coronavirus infection in children: experts' consensus statement. World Journal of Pediatrics.

12. Kunling Shen, Yonghong Yang, Tianyou Wang, Dongchi Zhao, Yi Jiang et al. (2020) Diagnosis, treatment, and prevention of 2019 novel coronavirus infection in children: experts' consensus statement. World Journal of Pediatrics.

13. KunLing Shen, Yong Hong Yang (2020) Diagnosis and treatment of 2019 novel coronavirus infection in children: a pressing issue. World Journal of Pediatrics.

14. (2019) Coronavirus disease 2019 (COVID-19), WHO, USA.

15. Chan JFW, Yuan S, Kok KH, To KKW, Chu H, et al. (2020) A familial cluster of pneumonia associated with the 2019 novel coronavirus indicating person-to-person transmission: a study of a family cluster. The Lancet 395(10223): 514-523.

16. Qi Lu,Yuan Shi (2020) Coronavirus disease (COVID-19) and neonate: What neonatologist need to know. J Med Virol.

ISSN: $2574-1241$

DOI: 10.26717/BJSTR.2020.26.004421

Mariano Cingolani. Biomed J Sci \& Tech Res

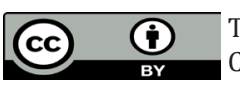

This work is licensed under Creative Commons Attribution 4.0 License

Submission Link: https://biomedres.us/submit-manuscript.php
17. (2019) Coronavirus, Gravidanza A Tincture with Ala Seno, Ila Wademekkum Daegli, Medicina E Ricerca.

18. Qi Lu, Yuan Shi (2020) Coronavirus disease (COVID-19) and neonate: What neonatologist need to know. J Med Virol.

19.ZhiMin Che, Zhi Min Chen, Jun Fen Fu, Qiang Shu, Ying Hu Chen, et al. (2020) Diagnosis and treatment recommendations for pediatric respiratory infection caused by the 2019 novel coronavirus. World Journal of Pediatrics.

20. Kunling Shen, Yonghong Yang, Tianyou Wang, Dongchi Zhao, Yi Jiang, et al. (2020) Diagnosis, treatment, and prevention of 2019 novel coronavirus infection in children: experts' consensus statement. World Journal of Pediatrics.

21. (2014) Amendments to the civil code regarding filiation 2014. Revision of the current provisions on filiation, in accordance with article 2 of the lawn: 219

22. Legge 22 dicembre (2017) Norme in materia di consenso informato e di disposizioni anticipate di trattamento. Official Gazzette Serie Generale $\mathrm{n}: 12$

23. Conti A, Capasso E, Casella C, Fedeli P, Salzano FA, et al. (2018) Blood transfusion in children: the refusal of Jehovah's Witness parents. Open Medicine 13(1): 101-104.

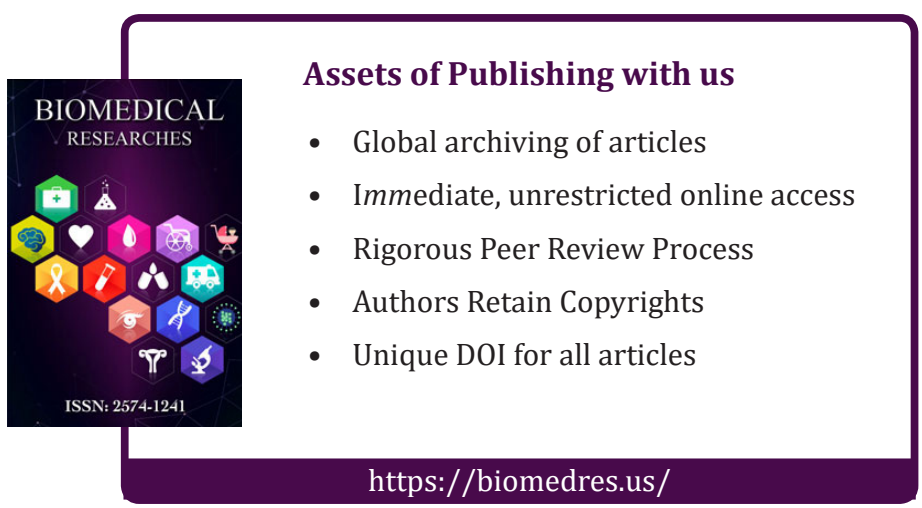

